Para enlazar con este artículo / To link to this article:

http://dx.doi.org/10.14198/fem.2018.31.01

Para citar este artículo / To cite this article:

Cerretti, Gabriella y Navarro-Guzmán, Capilla. «Análisis de las diferencias de género en cuanto a las relaciones sexuales y afectivas en parejas adolescentes». En Feminismo/s, 31 (junio 2018): 23-38. Dosier monográfico: Sexo y bienestar. Mujeres y diversidad, coords. Carmen Mañas Viejo y Alicia Martínez Sanz, DOI: 10.14198/fem.2018.31.01

\title{
ANÁLISIS DE LAS DIFERENCIAS DE GÉNERO EN CUANTO A LAS RELACIONES SEXUALES Y AFECTIVAS EN PAREJAS ADOLESCENTES
}

\section{ANALYSIS OF GENDER DIFFERENCES IN ADOLESCENTS' SEXUAL AND EMOTIONAL RELATIONSHIPS}

\author{
Gabriella CERRETTI \\ Project Manager at CISP \\ International Committee for the development of People, Roma (Italia) \\ orcid.org/0000-0003-0152-8483
Capilla NAVARRO-GUZMÁN
Universitat de les Illes Balears orcid.org/0000-0002-5805-0954

\section{Resumen}

Durante el proceso de socialización y a través de los diferentes agentes socializadores, aprendemos qué significa enamorarse, cómo debe ser la relación de pareja y también todos los mitos culturales sobre el amor. Estos contenidos sobre las relaciones amorosas son básicamente una transposición de los valores imperantes de la sociedad que nos rodea, es decir, sexistas y no igualitarios. El objetivo de esta investigación es analizar, desde una perspectiva de género, en qué medida estas creencias sobre la sexualidad siguen presentes en las relaciones entre adolescentes. A una muestra de 320 estudiantes de Bachillerato, se administró un cuestionario diseñado ad hoc. Los resultados indican diferencias en función de la variable sexo en referencia a: la pornografía, la sexualidad, el SIDA y la masturbación. Aunque la deseabilidad social introduce algunas limitaciones, los resultados sugieren la importancia de introducir en las escuelas una educación afectiva y sexual con la finalidad de trasmitir valores 
y actitudes que favorezcan el respeto y en contra del sexismo en cualquiera de sus manifestaciones.

Palabras claves: adolescentes, sexualidad, violencia de género, relación de pareja.

\begin{abstract}
During the process of socialization and by the different socialization agents, we learn what falling in love means, how a couple relationship should be and all myths about love as well. These contents about love relationships basically are a transposition of the leading values of the surrounding society, which is sexist and no equal. The objective of this study is to analyze through a gender perspective in which measure the beliefs about sexuality persist in the adolescents dating. 320 high school students of High School, answered a questionnaire specially prepared for this purpose. Results show significant gender differences about: pornography, sexuality, AIDS and masturbation. Although social desirability presents some limits, these results show the importance of introduction in schools the sexual and emotional education, to transmit values and attitudes against sexism.
\end{abstract}

Keywords: adolescents, sexuality, gender-based violence, dating.

\title{
1. INTRODUCCIÓN
}

La socialización es un proceso de aprendizaje que se inicia en el momento del nacimiento y perdura durante toda la vida, a través del cual las personas en interacción con otras personas, aprenden e interiorizan los valores, las actitudes, las expectativas y los comportamientos característicos de la sociedad en la que han nacido y que le permiten desenvolverse en ella (Giddens 85). Según la teoría de la socialización diferencial, las personas, en su proceso de iniciación a la vida social y cultural, y a partir de la influencia de los agentes socializadores (como la familia, la escuela, el grupo de pares, solo para ofrecer algunos ejemplos), adquieren identidades diferenciadas de género que conllevan estilos cognitivos, actitudinales, conductuales y morales, y normas estereotípicas de la conducta asignada a cada género (Barton y Walker 12). Esta socialización diferencial afecta a muchos y diversos aspectos de la vida humana y, entre ellos, a las relaciones afectivas y de pareja.

Así, durante el proceso de socialización entre muchas otras cosas, aprendemos qué significa enamorarse, qué sentimientos son apropiados, de quién 
debemos o no enamorarnos, qué o quién es atractivo (lo cual suele coincidir con los patrones de los roles de género tradicionales), cómo debe ser la relación de pareja y, evidentemente, también todos los mitos culturales sobre el amor (Duque 108; Flecha, Puigvert, y Redondo 112).

Los contenidos sobre las relaciones interpersonales, amorosas y de pareja que nos llegan a través de los diferentes agentes socializadores, son básicamente una transposición de los valores imperantes de la sociedad que nos rodea, es decir, sexistas y no igualitarios, como muestran diversas investigaciones sobre el tema (Altable 198; Charkow y Nelson 14; Oliver y Valls 95; Mañas et al. 9; Moreno, González, y Ros 22; Rodríguez-Castro, Lameira y Carrera 12). Consideramos que analizar las relaciones de pareja que se crean en la adolescencia es crucial porque en esas primeras relaciones amorosas es donde van a formarse sus ideas iniciales sobre qué esperar de una relación de pareja y cómo comportarse en la intimidad, algo que va a repercutir en su vivencia de la etapa adulta. Así, Flecha et al. (112) enmarcan su estudio en la actuación preventiva contra la violencia de género desde el ámbito social y de la educación, apostando por la intervención en los procesos de socialización y en la educación afectiva y sexual. En dicho estudio se resalta la importancia de la socialización como forma de transmisión de un imaginario social de las relaciones afectivo-sexuales que atribuyen valores positivos y negativos a determinadas formas de relación entre hombres y mujeres, a través de la creación de estereotipos que construyen socialmente lo que se nos presenta como atractivo y lo que no. Por imaginario social nos referimos a un fenómeno que da cuenta de un ideario creado a partir de la acción de los individuos en tanto que seres en conexión directa con su entorno socio-cultural (Castoriadis 480). Por lo tanto el imaginario social no responde a conceptos, ni a categorías trascendentales, ni a la razón lógica, el imaginario alude a una ficción vivida por los individuos en un determinado momento histórico y proyecta el entorno socio-cultural de esa época. Así, partiendo de la base de que el amor es un constructo social y no un fenómeno originado en causas biológicas o químicas, se precisa el desarrollo de una nueva socialización o resocialización de nuestro concepto de amor, de los modelos de amor que consideramos deseables y aceptables y de las relaciones afectivas y sexuales como herramienta fundamental para la prevención y erradicación de la violencia de género (Boch, Ferrer y Alzamora 28). 
En este sentido, Isabel Barrera (4) remarca que los seres humanos no nacen violentos, sino que la violencia es una cualidad que se va adquiriendo a medida que el ser humano se va integrando en la sociedad y va asimilando los patrones establecidos. Por ello estamos en la obligación de educar a chicos y chicas en reconocer las acciones violentas, no solo físicas sino también psicológicas y saber actuar ante estas situaciones con la finalidad de motivarlos a cambiar la realidad en la cual vivimos.

Con este trabajo que se enmarca en una investigación más amplia, pretendemos examinar las relaciones afectivo-sexuales entre adolescentes y detectar si en estas primeras relaciones existen diferencias en función de la variable sexo que podrían dar lugar a situaciones discriminatorias y de violencia contra las mujeres, problema social de máxima relevancia que tan devastadoras consecuencias puede llegar a tener para la vida y la salud de todas las personas (Calvete, Estevez y Corral 448; Sarasúa et al. 460).

Más en detalle pretendemos analizar la actitud que los chicos y las chicas tienen hacia la sexualidad, el SIDA, la masturbación y qué nivel de conocimiento tienen del propio cuerpo. El análisis ha sido realizado en función de variables socio-demográficas y desde la perspectiva de género, evaluando pues en qué medida la conducta adoptada obedece a motivaciones diferentes entre las chicas y los chicos. Con respecto a la edad de la muestra (entre 16 y 18 años), se ha decidido focalizar este trabajo en la etapa de la adolescencia y en los primeros contactos íntimos, ya que es crucial a la hora del desarrollo del sexismo que personas adolescentes aprendan a identificar los comportamientos sexistas y las desventajas que estos conllevan tanto para los chicos como para las chicas (Montañés et al. 223).

\section{MÉTODO}

\subsection{Participantes}

La muestra del estudio está compuesta por 320 estudiantes de Bachillerato ( $48 \%$ chicos y $52 \%$ chicas). La mayoría de las personas encuestadas indica que su nacionalidad es de un país europeo $(83,1 \%)$, donde España es el país de origen más representado (96,1\%); sigue América Latina con un 14,2\%, siendo Ecuador el país de donde proviene la mayoría del alumnado latinoamericano (20\%). 


\subsection{Instrumentos}

Para recoger datos sobre las variables socio-demográficas se empleó un cuestionario diseñado ad hoc que incluía información relativa a sexo, lugar de residencia y país de nacimiento. También se preguntó si en el momento de la encuesta la persona encuestada tenía pareja o si no la había tenido nunca, y en el caso de tener pareja, se pedía que especificara el sexo de la misma (chico/chica). No se pedían informaciones referentes a la diversidad sexual ni a las entidades transgénero porque nuestro estudio pretendía profundizar acerca de la violencia de género en parejas heterosexuales solamente. En cuanto a las creencias y prejuicios acerca de la sexualidad, se optó por utilizar la Batería Exploratoria de Sexualidad (BES-III. Actitudes) de Ballester y Gil (189): una herramienta que analiza los mitos y las creencias sobre la sexualidad en relación a la actividad sexual, la homosexualidad, la pornografía, la masturbación, la reproducción, el sexo oral y el SIDA. Come se puede apreciar en la tabla 1, consiste en un listado de doce ítems a los que se contesta en formato dicotómico (sí/no).

Tabla I. Batería Exploratoria de Sexualidad (BES-III. Actitudes): listado de doce ítems a los que se contesta en formato dicotómico

\begin{tabular}{|c|c|c|}
\hline 1. La homosexualidad es una característica con la que se nace & sí & no \\
\hline $\begin{array}{l}\text { 2. La pornografía tiene efectos perjudiciales en la sexualidad de una } \\
\text { persona }\end{array}$ & sí & no \\
\hline 3. No hay mujeres frígidas sino hombres inexpertos & sí & no \\
\hline 4. La masturbación es una forma de ser infiel a la pareja & Sí & no \\
\hline $\begin{array}{l}\text { 5. Está bien que las personas de edad avanzada sigan teniendo } \\
\text { actividad sexual }\end{array}$ & sí & no \\
\hline $\begin{array}{l}\text { 6. La sexualidad es una faceta humana que nos acompaña desde el } \\
\text { nacimiento }\end{array}$ & sí & no \\
\hline 7. El fin principal de la sexualidad es la reproducción & sí & no \\
\hline $\begin{array}{l}\text { 8. La atracción sexual hacia personas de sexo opuesto tiene un origen } \\
\text { biológico }\end{array}$ & sí & no \\
\hline 9. La sexualidad sana es la que se realiza con la persona amada & sí & no \\
\hline $\begin{array}{l}\text { 10. El consumo de material pornográfico incita a la violación y otros } \\
\text { delitos sexuales }\end{array}$ & sí & no \\
\hline $\begin{array}{l}\text { 11. El sexo oral y la masturbación mutua en la pareja son síntomas de } \\
\text { inmadurez y neurosis }\end{array}$ & sí & no \\
\hline 12. El SIDA es consecuencia de una vida sexual promiscua y pervertida & sí & no \\
\hline
\end{tabular}

Feminismo/s 31, junio 2018, pp. 23-38 
Finalmente, para analizar el nivel de conocimiento que chicos y chicas tienen de su propio sexo, así como las diferencias entre unos y otras en cuanto a la práctica de la masturbación, se presentaron dos ítems de elaboración propia que permitían una respuesta dicotómica (Sí/No), concretamente: ¿Consideras que conoces bien tus partes intimas y tus zonas erógenas? y ¿Practicas la masturbación?

\subsection{Procedimiento}

Previamente a las visitas en los institutos, se solicitó la autorización de acceso a las aulas a través de una carta, enviada por correo electrónico, donde se explicitaba que el trabajo estaba avalado por el Instituto de Ciencias de la Educación (ICE) de la Universidad de las Islas Baleares (UIB) y por la Consejería de Educación, organismos que facilitaron un permiso especial para poder realizar la investigación. El cuestionario descrito fue autoaplicado y se pasó de manera colectiva antes de empezar cada clase o en la hora de tutoría. Se solicitó su participación al alumnado de manera voluntaria y se garantizó su anonimato. El tiempo medio dedicado a administrar el cuestionario fue de unos 20-30 minutos en cada grupo.

\subsection{Análisis de los datos}

Para analizar los datos recopilados, dada la naturaleza de las variables, se ha aplicado la prueba de independencia ji-cuadrado de Pearson, mediante el programa informático SPSS v. 14.0.

\section{RESULTADOS}

Del total de las doce preguntas contenidas en la Batería Exploratoria de la Sexualidad (BES-III. Actitudes), cuatro de ellas han mostrado diferencias estadísticamente significativas entre hombres y mujeres. En primer lugar, y como indicamos en la siguiente tabla (tabla 2), se han hallado diferencias entre alumnos y alumnas en cuanto al tema de la pornografía: «la pornografía tiene efectos perjudiciales en la sexualidad de una persona» (ítem 2): aquí las chicas han contestado de forma afirmativa en mayor proporción que los chicos con $35,6 \%$ frente a un $19,9 \%$. 
Tabla II. Análisis de la relación entre el ítem 2 de la BES-III y el sexo

\begin{tabular}{|c|c|c|c|c|}
\hline BES-III: Ítem 2 & Chicos & Chicas & $\mathrm{X}^{2}$ & Signif. \\
\cline { 1 - 3 } Sí & $19.9 \%$ & $35.6 \%$ & \multirow{2}{*}{9.597} & $<.005$ \\
\cline { 1 - 3 } No & $80.1 \%$ & $64.4 \%$ & & \\
\hline
\end{tabular}

$\mathrm{N}=314$, g.l.=1

Otro aspecto objeto de análisis sobre la sexualidad en el que se han observado diferencias entre chicos y chicas, es la opinión del alumnado frente la afirmación «el fin principal de la sexualidad es la reproducción» (ítem 7), que recoge más desacuerdo entre las chicas con un $83,6 \%$, superando a los chicos con un $67,1 \%$ (ver tabla 3 ).

Tabla III. Análisis de la relación entre el ítem 7 de la BES-III y el sexo

\begin{tabular}{|c|c|c|c|c|}
\hline BES-III: Ítem7 & Chicos & Chicas & $\mathrm{X}^{2}$ & Signif. \\
\hline Sí & $32.9 \%$ & $16.4 \%$ & \multirow{2}{*}{11.757} & $<.005$ \\
\hline No & $67.1 \%$ & $83.6 \%$ & & \\
\hline
\end{tabular}

$\mathrm{N}=317$, g.l.=1

También en referencia a la afirmación «el consumo de material pornográfico incita a la violación y otros delitos sexuales» (ítem 10), se observan diferencias con significación estadística. Así, como se indica en la tabla que presentamos a continuación (tabla 4), las chicas han manifestado estar más de acuerdo contestando afirmativamente con un 28,2\%, frente al $17,6 \%$ de los chicos.

Tabla IV. Análisis de la relación entre el ítem 10 de la BES-III y el sexo

\begin{tabular}{|c|c|c|c|c|}
\hline BES-III: Ítem 10 & Chicos & Chicas & $\mathrm{X}^{2}$ & Signif. \\
\hline Sí & $17.6 \%$ & $28.2 \%$ & \multirow{2}{*}{4.967} & $<.005$ \\
\hline No & $82.4 \%$ & $71.6 \%$ & & \\
\hline
\end{tabular}

$\mathrm{N}=316$, g.l.=1

Feminismo/s 31, junio 2018, pp. 23-38 
Se observan diferencias en función de la variable sexo también con el ítem 12: «el SIDA es consecuencia de una vida sexual promiscua y pervertida», donde del total de chicos que han contestado a esta pregunta, el 33,1\% responde de manera afirmativa, frente al $22,8 \%$ de las chicas (ver tabla 5).

Tabla V. Análisis de la relación entre el ítem 12 de la BES-III y el sexo

\begin{tabular}{|c|c|c|c|c|}
\hline BES-III: Ítem 12 & Chicos & Chicas & $\mathrm{X}^{2}$ & Signif. \\
\cline { 1 - 3 } Sí & $33.1 \%$ & $22.8 \%$ & \multirow{2}{*}{4.110} & $<.005$ \\
\hline No & $66.9 \%$ & $77.2 \%$ & & \\
\hline
\end{tabular}

$\mathrm{N}=313$, g.l.=1

Referente a los dos ítems de elaboración propia, en ambos se observan diferencias con significación estadística. Así, frente a la primera pregunta «¿Consideras que conoces bien tus partes íntimas y tus zonas erógenas?» se observan diferencias estadísticamente significativas entre hombres y mujeres en cuanto al nivel de conocimiento de sus zonas erógenas (ver tabla 6). Concretamente, del total de chicos que han contestado a esta pregunta, el 88,3\% (136 varones) responde de manera afirmativa, mientras que las chicas contestan afirmativamente en menor proporción, con un 62,8\% (103 mujeres).

Tabla VI. Análisis de la relación entre el ítem 1 de elaboración propia y el sexo

\begin{tabular}{|c|c|c|c|c|}
\hline $\begin{array}{c}\text { Ítem 1 } \\
\text { (elaboración } \\
\text { propia) }\end{array}$ & Chicos & Chicas & $\mathrm{X}^{2}$ & Signif. \\
\cline { 1 - 3 } Sí & $88.3 \%$ & $62.8 \%$ & \multirow{2}{*}{27.674} & $<.001$ \\
\hline No & $11.7 \%$ & $37.2 \%$ & \\
\hline
\end{tabular}

$\mathrm{N}=318$, g.l.=1

También en referencia a la práctica de la masturbación («iPracticas la masturbación?»), el análisis estadístico nos muestra que se dan diferencias estadísticamente significativas entre hombres y mujeres al responder a la pregunta planteada. Así, si nos fijamos en los porcentajes de alumnos y alumnas 
que han afirmado practicar la masturbación, ellos superan a sus compañeras con un $87 \%$ frente a un $27,8 \%$ (ver tabla 7 ).

Tabla VII. Análisis de la relación entre el ítem 2 de elaboración propia y el sexo

\begin{tabular}{|c|c|c|c|c|}
\hline $\begin{array}{c}\text { Ítem 2 } \\
\text { (elaboración } \\
\text { propia) }\end{array}$ & Chicos & Chicas & $\mathrm{X}^{2}$ & Signif. \\
\hline Sí & $87.0 \%$ & $27.8 \%$ & \multirow{2}{*}{112.800} & $<.001$ \\
\hline No & $13.0 \%$ & $72.2 \%$ & \\
\hline
\end{tabular}

$\mathrm{N}=316$, g.l.=1

\section{DISCUSIÓN Y CONCLUSIONES}

Los resultados obtenidos permiten concluir que hombres y mujeres parecen responder de forma diferente ante los siguientes temas: la pornografía, el fin principal de la sexualidad, el SIDA, la actitud hacia la masturbación y los conocimientos del propio cuerpo. Referente a la pornografía, aunque pareciera que las mujeres consumen menos pornografía, esto no es un hecho evolutivo ni natural, sino simplemente cultural (Figari 182). De hecho, como apunta Kinsey (citado por Mantecón 203), las pulsiones sexuales no entienden de género y no hay diferencias fisiológicas para sentir placer más allá de las preferencias personales. Pero, desde luego, lo que sí existe son una serie de condicionantes culturales determinantes para nuestra prácticas y comportamientos sexuales y no es de extrañar, por lo tanto, que también la pornografía y su uso reflejen sesgos de género.

En referencia al fin principal de la sexualidad y en consonancia con nuestros resultados, estudios anteriores (INJUVE 22; Laqueur 95) indican que, de forma global, los y las jóvenes atribuyen a la sexualidad una función comunicativa-placentera, considerándola como un medio para el equilibrio personal, la comunicación con el otro/a y el placer compartido. Resulta, por el contrario, minoritaria la concepción reproductiva tradicional donde el sexo aparecía ceñido por completo a la biología, siendo comprendido únicamente como un instrumento para tener hijos/as. 
Referente a los prejuicios acerca del SIDA, en España desde la aparición de los primeros casos de esta enfermedad, se estableció un paralelismo entre este virus y una identidad sexual concreta, que propició un concepto generalizado del SIDA como enfermedad del otro, con los consecuentes prejuicios y estigmas hacia las personas afectadas (Martín 242). Cierto es que el inicio de esta enfermedad fue un tanto espectacular y se dio más entre homosexuales, elemento que dio lugar a la idea errónea que se trataba de una enfermedad de gays y de personas drogadictas (Aguete 10). Actualmente los conocimientos acerca del SIDA han variado considerablemente, pero aunque ahora se sabe que todos y todas somos susceptibles de ser infectados e infectadas, los antecedentes históricos de la enfermedad y la cultura discriminatoria han provocado la persistencia del estereotipo que identifica todavía al SIDA como una enfermedad de homosexuales, lo cual alimenta la falsa creencia de que teniendo únicamente relaciones heterosexuales no existe riesgo de contagio (Espada, Quiles y Méndez 11). A este respecto hay que recordar que las campañas estatales en España, sobre todo las primeras alrededor de los años 90, hacían una referencia implícita, en la mayoría de las ocasiones, a la monogamia, y daban a entender que cuanto mayor era el grado de promiscuidad, mayor era el riesgo de contraer la enfermedad. Lo cierto es que, en realidad, si todas las relaciones sexuales son practicadas con preservativo, las posibilidades de contagio por vía sexual son igual de escasas. Pero la difusión de conceptos erróneos y moralistas en el transcurso de los últimos veinte años, ha dado lugar a la proliferación de falsos mitos acerca del SIDA que, como se ha visto en los resultados del estudio, siguen presentes, aunque en bajos porcentajes. Así, existe todavía la creencia de que los hombres heterosexuales se consideran ajenos a este virus por pensar que es una enfermedad propia de los homosexuales. Y si para unos el SIDA les es ajeno, para otros les es imposible adoptar medidas de prevención por el miedo de que se piense que eso plantea la posibilidad de que se esté en una condición de riesgo, debido a una supuesta preferencia sexual (Itzel y López 8).

Referente al conocimiento del propio cuerpo, la práctica de la masturbación y la sexualidad con la pareja, nuestros resultados, en línea con los de otros estudios (Palenzuela 460; Estarli 41), indican que los y las adolescentes presentan bajos conocimientos y creencias erróneas en materia de sexualidad. Sobre todo, en referencia a la masturbación femenina, es significativo 
el desconocimiento que en general se tiene sobre esta práctica, casi como si perteneciera solo al mundo sexual masculino.

Interesante también reflexionar sobre otro de los temas analizados: la forma de concebir la sexualidad. Hemos visto que hay bastante desacuerdo tanto en las chicas $(83,6 \%)$ como en los chicos $(67,1 \%)$ frente la afirmación «el fin principal de la sexualidad es la reproducción» y este resultado podría llevar a pensar que la gran mayoría de jóvenes se adhiere a una concepción de la sexualidad comunicativa/placentera, lejos de una concepción puramente reproductiva. Sin embargo, a pesar de esta consideración, nos encontramos con unos datos un poco contradictorios si relacionamos el tema del conocimiento del propio cuerpo, con la actitud hacia la masturbación y con la concepción de la sexualidad: así los resultados nos indican que el 62,8\% de las chicas encuestadas afirma conocer sus zonas erógenas y tan solo un 27,8\% practica la masturbación.

Mirando más en conjunto estos temas y considerando que hay acuerdo en la importancia del conocimiento del propio cuerpo y de las partes erógenas como base para vivir de forma satisfactoria la sexualidad tanto de forma individual como con otra persona (Geer y Robertson 673; Nobre et al. 368; Ortega et al. 271; Perla et al. 12; Sueiro, Diéguez y Chas 102; Trudel 231), nos surgen unas reflexiones espontáneas: si un 62,8\% de las chicas encuestadas afirma conocer las zonas de su cuerpo donde buscar y encontrar el placer, ¿Cómo es posible que sólo un 27,8\% indique que practica la masturbación? Además, ¿cómo puede una persona afirmar conocer su cuerpo si no lo explora? Y finalmente, ¿cómo se puede tener una sexualidad placentera, si no se conocen las zonas del propio cuerpo que custodian el placer sexual? La aparente incongruencia de los resultados obtenidos nos puede llevar a concluir que, por deseabilidad social o vergüenza, quizás algunas personas pueden no querer reconocer que se masturban y precisamente esto puede ocurrir sobre todo entre las chicas.

También Barberá y Navarro (68) en su trabajo sobre la construcción de la sexualidad en la adolescencia, confirman que las chicas tienen un escaso interés por este tema y no se masturban hasta más tarde, cuando ya han tenido alguna experiencia erótica con un chico. Según las autoras «la diferencia entre sexos en la práctica de la masturbación estaría organizando parte de la asimetría en relación con la sexualidad que viven chicos y chicas» (74). Cabe 
entonces preguntarse ¿por qué las chicas esperan a practicar el autoerotismo hasta haber tenido relaciones sexuales? La respuesta a este interrogante no es de carácter biológico, ya que como indica Kervasdoué (102) los cambios puberales se accionan en las chicas alrededor de un año y medio antes que en ellos. Entonces, siendo sexualmente más maduras, ¿por qué son sexualmente menos eróticas? La conclusión a la cual llegan las autoras es que, de nuevo, será un sistema de creencias y representaciones sociales lo que opera a esta edad, cuyo fin fundamental será el control y la subordinación de la sexualidad de las chicas. Así, en la construcción de la sexualidad femenina, la idea del sexo como placer estaría relacionado al concepto de relación de pareja y de interdependencia afectiva con el otro, relegando el goce sexual hasta el momento del intercambio amoroso. La masturbación, por lo tanto, no podría formar parte fácilmente de la sexualidad de las chicas que aún no han tenido relaciones sexuales con un chico, subrayando una vez más una condición erótica de dependencia hacia el otro.

En definitiva, volviendo a nuestros resultados, tanto en el caso que las chicas encuestadas hayan dicho la verdad, como en el caso de que no, se puede afirmar que existe una cierta incomodidad a la hora de tratar el tema de la masturbación, y se considera por lo tanto necesario insistir en una educación afectiva y sexual que aclare dudas donde las haya y favorezca cambios de mentalidad y actitud para conseguir una sexualidad libre de prejuicios. Se necesita fundamentalmente sensibilizar a la comunidad educativa para propiciar un cambio de comportamientos y contenidos, encaminados a erradicar el sexismo y la violencia de género en la sociedad. Creemos por lo tanto que la escuela, con su papel de agente socializador, representa un motor de cambio fundamental porque ofrece un espacio muy adecuado para la reflexión individual y colectiva sobre los problemas analizados, las causas que los producen y las consecuencias que acarrean.

Para finalizar, debemos señalar que, si bien este trabajo ofrece información que creemos puede ser relevante sobre algunas características del alumnado de bachillerato de Mallorca, a partir de una muestra significativa del total de estudiantes, no está exento de algunas limitaciones. En primer lugar, queremos destacar las dificultades que encontramos para poder acceder a las aulas y entregar la encuesta al alumnado. En detalle, de los sesenta y tres institutos con los que contactamos por correo electrónico y después telefónicamente,

Feminismo/s 31, junio 2018, pp. 23-38 
conseguimos visitar solo nueve. En la mayoría de los casos no recibimos respuestas a nuestros e-mails y a la hora de llamar por teléfono, tuvimos muchas dificultades para poder conseguir hablar con las personas adecuadas.

Queremos además añadir que registramos, por lo general, muy poco interés por parte de la Dirección de los institutos en colaborar a este tipo de actividad. Al respecto, destacamos también que dos institutos concertados, dirigidos por personal religioso, nos contestaron que no estaban interesados en participar en la investigación por el tema tratado y un instituto público no quiso participar porque el cuestionario estaba escrito en castellano y no en catalán. A pesar de haber contestado que, aunque quisiéramos, no podíamos traducir el instrumento al catalán, porque las escalas se tienen que utilizar en el idioma en el que fueron validadas y fueron validadas en castellano, la dirección del centro negó su colaboración.

Entre las limitaciones del estudio, es importante tener en cuenta también que parte del cuestionario estaba basado en la autodeclaración de los comportamientos sexuales, por lo que la validez de las respuestas es limitada, pudiendo dar lugar a cierto sesgo de información. No obstante, consideramos que el anonimato de los cuestionarios favorece una mayor sinceridad y al respecto, cabe destacar también que las particularidades de los estudios sobre temas de sexualidad siempre plantean la posibilidad de sesgos de deseabilidad social en las personas que participan en las encuestas a la hora de ofrecer las respuestas (de la Barrera 40).

De manera global consideramos que los desequilibrios en las relaciones de parejas representan una problemática actual que requiere insistir con investigaciones que profundicen en las creencias de la adolescencia sobre el amor y las relaciones afectivo-sexuales como medida preventiva a la violencia género.

\section{REFERENCIAS BIBLIOGRÁFICAS}

Aguete, Concepción. «Educación sexual y SIDA: propuesta educativa para los centros de educación secundaria». Revista Digital Transversalidad Educativa 22 (2009): 4-14.

Altable, Charo. Penélope o las trampas del amor. Valencia: Nau, 1998.

Ballester, Rafael y Gil, M. ${ }^{a}$ Dolores. «Salud sexual (II): studio de actitudes sexuales en nuestro contexto». Análisis y modificación de conducta 23 (1997): 181-209. 
Barberá, Esther y Navarro, Esperanza. «La construcción de la sexualidad en la adolescencia». Revista de Psicología Social 15. 1 (2000): 63-75.

Barrera, Isabel. «Como trabajar la violencia de género en el aula». Revista digital Innovación y Experiencias Educativas (2009). https://es.scribd.com/document/275320664/Isabel-Barrera-Benitez02, último acceso 6 de noviembre 2017.

Barton, Len; Walker, Stephen y Westhill Sociology of Education Conference. Gender, Class \& Education. Lewes: Falmer Press, 1983.

Bosch, Esperanza; Ferrer, Victoria y Alzamora, Aina. El laberinto patriarcal. Barcelona: Antrophos, 2006.

Calvete, Esther; Estevez, Ana y Corral, Susana. «Trastornos por estrés postraumático y su relación con esquemas cognitivos disfuncionales en mujeres maltratadas». Psicothema 19(3) (2007): 446-451.

Castoriadis, Cornelius. La institución imaginaria de la sociedad. Traducción del francés al español de Antoni Vicens y Marco Aurelio Galmarini. (2. ${ }^{a}$ reimpresión). Buenos Aires Argentina: Fábula TusQuets Editores, 2003.

Charkow, Wendy y Nelson, Eileen. «Relationship dependency, dating violence and scripts of female». Journal of College Counselling 3.1 (2000): 12-17.

De la Barrera, Citlalli Pérez. «Habilidades para la vida y uso de anticoncepción por tipo de pareja sexual en adolescentes». Enseñanza e Investigación en Psicología 18.1 (2013): 35-49.

Duque, Elena. ¿Aprendiendo para el amor o para la violencia? Las relaciones en las discotecas. Barcelona: El Roure, 2006.

Espada, José Pedro; Quiles, M. ${ }^{a}$ José y Méndez, Francisco Javier. «Conductas sexuales de riesgo y prevención del sida en la adolescencia». Papeles del psicólogo (2003). http://www.papelesdelpsicologo.es/vernumero.asp?id=1076, último acceso 6 noviembre 2017.

Estarli, Rafael. «Programa de educación sexual y afectiva: una experiencia educativa del proyecto Granada ciudad educadora». Revista de Estudios de Juventud 63 (2003): 37-47.

Figari, Carlos Eduardo. «Placeres a la carta: consumo de pornografía y constitución de géneros». La ventana. Revista de estudios de género 3.27 (2008): 170-204.

Flecha, Ainhoa; Puigvert, Lidia y Redondo, Gisela. «Socialización preventiva de la violencia de género». Feminismo/s 6 (2005): 107-120. 
Geer, James y Robertson, Gloria. «Implicit attitudes in sexuality: Gender differences». Archives of Sexual Behavior, 34.6 (2005): 671-677.

Giddens, Anthony. La transformación de la intimidad. Sexualidad, amor y erotismo en las sociedades modernas. Madrid: Cátedra, 1995.

INJUVE. Informe de la Juventud en España 2012. http://www.injuve.es/observatorio/demografia-e-informacion-general/informe-de-la-juventud-en-espana-2012, 2013, último acceso 6 noviembre 2017.

Itzel, Helena y López, Samantha. «Actitud de los de los estudiantes de sexo masculino de la carrera de Psicología de la Universidad Iberoamericana hacia las Personas con VIH/ SIDA». AMAPSI. Asociación Mexicana de Alternativas en Psicología, 2014. http://www.amapsi.org/portal/index2.php?option=com_ content\&do_pdf=1\&id=157, último acceso 6 noviembre 2017.

Kervasdoué, Anne. Cuestiones de mujeres. Madrid: Alianza, 1995.

Laqueur, Thomas. La construcción del sexo. Madrid: Cátedra, 1994.

Mantecón, Marta. «Visiones de lo invisible: mujeres creando y girls who like porno». En D’Agostino, D., Pacheco, V., Torres, K. y Viñuela, E. (eds.), Feminismo e Interculturalidad. Sevilla: ArCiBel Editores, 2008.

Mañas, Carmen; Martínez-Sanz, Alicia., Esquembre, Mar., Montesinos, Nieves y Gilar, Raquel. «Exploración de la violencia en las relaciones de pareja de jóvenes universitarias/os». Centro de Estudios sobre la Mujer (CEM), Universidad de Alicante, 2012. http://m.web.ua.es/en/ice/jornadas-redes-2012/documentos/posteres-expuestos/245605.pdf, último acceso 6 noviembre 2017.

Martín, Rut. «El SIDA ante la opinión pública: el papel de la prensa y las campañas de prevención estatales en la representación social del SIDA en España». Studium: Revista de humanidades 15 (2009): 237-268.

Montañés, Pilar; Megías, Jesus, De Lemus, Soledad y Moya, Miguel. «Influence of early romantic relationships on adolescents' sexism/Sexismo en la adolescencia: influencia de las primeras relaciones de pareja». Revista de Psicología Social 30.2 (2015): 219-240.

Moreno, Montserrat; González, Alba y Ros, Marc. «Enamoramiento y violencia contra las mujeres». En V.A. Ferrer y E. Bosch (eds.), Los feminismos como herramientas de cambio social (II): de la violencia contra las mujeres a la construcción del pensamiento feminista. Palma de Mallorca: UIB, 2007.

Nobre, Pedro; Wiegel, Marcus, Bach, Amy, Weisberg, Risa, Brown, Timothy, Wincze, John y Barlow, David. «Determinants of sexual arousal and the 
accuracy of itself-estimation in sexually functional males». Journal of Sex Research 41.4 (2004): 363-371.

Oliver, Esther y Valls, Rosa. Violencia de género. Investigaciones sobre quiénes, por qué y cómo superarla. Barcelona: Roure, 2004.

Ortega, Virgilio; Ojeda, Pilar, Sutil, Francisca y Sierra, Juan Carlos. «Culpabilidad sexual en adolescentes: Estudio de algunos factores relacionados». Anales de Psicología 21.2 (2005): 268-275.

Palenzuela, Ángeles. «Intereses, conducta sexual y comportamientos de riesgo para la salud sexual de escolares adolescentes participantes en un programa de educación sexual». Análisis y Modificación de Conducta 32.144 (2006): 451-495.

Perla, Felipe; Sierra, Juan Carlos, Vallejo, Pablo y Gutiérrez-Quintanilla, Ricardo (2009). «Un estudio psicométrico de la versión española reducida del Hurlbert Index of Sexual Fantasy». Boletín de Psicología 96 (2009): 7-16.

Rodríguez-Castro, Yolanda; Lameiras, María y Carrera, María Victoria. «Amor y Sexismo: una peligrosa relación en los y las adolescentes gallegos/as». Revista de Estudios e Investigación en Psicología y Educación 2 (2015): 011-014.

Sarasua, Belen; Zubizarreta, Irene, Echeburúa, Enrique y De Corral, Paz. «Perfil psicopatológico diferencial de las víctimas de violencia de pareja en función de la edad». Psicothema 19.3 (2007): 459-466.

Sueiro, Encarnación; Diéguez, Jose Luis y Chas, M. ${ }^{a}$ Dolores. «Primeras fuentes de información, conocimientos, actitudes y prácticas sexuales. Su interrelación». Cuadernos de Medicina Psicosomática y Psiquiatría de Enlace 69/70 (2004): 95-114.

Trudel, Gilles. «Sexuality and marital life: Results of a survey». Journal of Sex $\mathcal{E}$ Marital Therapy 28.3 (2002): 229-249. 